\title{
Acoustic modes in metallic nanoparticles: atomistic versus elasticity modeling
}

\author{
Nicolas Combe* \\ CNRS ; CEMES (Centre d'Elaboration des Matériaux et d'Etudes Structurales); BP 94347, \\ 29 rue J. Marvig, F-31055 Toulouse, France. and \\ Université de Toulouse ; UPS ; F-31055 Toulouse, France \\ Lucien Saviot \\ Institut Carnot de Bourgogne, Université de Bourgogne, UMR 5209 CNRS, \\ 9 Avenue A. Savary, BP 47870, F-21078 Dijon Cedex, France
}

(Dated: June 30, 2009)

\begin{abstract}
The validity of the linear elasticity theory is examined at the nanometer scale by investigating the vibrational properties of silver and gold nanoparticles whose diameters range from about 1.5 to $4 \mathrm{~nm}$. Comparing the vibration modes calculated by elasticity theory and atomistic simulation based on the Embedded Atom Method, we first show that the anisotropy of the stiffness tensor in elastic calculation is essential to ensure a good agreement between elastic and atomistic models. Second, we illustrate the reduction of the number of vibration modes due to the diminution of the number of atoms when reducing the nanoparticles size. Finally, we exhibit a breakdown of the frequency-spectra scaling of the vibration modes and attribute it to surface effects. Some critical sizes under which such effects are expected, depending on the material and the considered vibration modes are given.
\end{abstract}

PACS numbers: 63.22.-m, 63.22.Kn,81.05Bx

\section{INTRODUCTION}

Describing structural and elastic properties at the nanometer scale has recently raised the interest of many scientists. Especially, the applicability of the linear elastic theory is questioned at these scales. Various approaches can be used to test the validity of linear elasticity. Different groups have tried to measure the elastic properties, most often the Young modulus, by mechanical deformation while decreasing the system size 1, 2, 3, 4, 5]. Another approach to probe the elastic properties uses acoustic vibration of nanoparticles (NP) and is mainly based on light or neutron scattering. Acoustic vibration modes frequencies of NP measured by Raman scattering or pump-probe experiments are fairly well reproduced by linear elastic theory calculations even for NPs with sizes of a few nanometers $6,6,7,8,9]$.

The present work is concerned with the validity of elasticity within the vibration properties computed for systems whose sizes are smaller than the ones currently and experimentally explored. To achieve this goal, we compare the vibration modes calculated by linear elasticity and atomistic semi-empirical potential calculations in the case of metallic NP of diameter ranging from 1.4 to about $4 \mathrm{~nm}$. The choice of metallic NP is motivated by the interest they arouse among the physicists community due to their plasmon properties opening many promising applications 10, 11].

Vibration properties of metallic NPs have been the

\footnotetext{
*Electronic address: combe@cemes.fr

†Electronic address: lucien.saviot@u-bourgogne.fr
}

subject of many theoretical studies using the linear elasticity or atomistic simulation, and experiments.

Since the nineteenth century, the linear elasticity has been used to calculate the vibrational modes of spherical particles [12]: such calculations generally agree well with experimental measurements of NP vibrations [7, 13]. Different refinements of this model have been performed taking into account the anisotropy of the stiffness tensor, the non-spherical shapes of the nanoparticles and matrix effects [14, 15, 16]. The improvements of the experimental energy resolution and of the synthesis of such NPs have allowed to demonstrate subtle effects due to the anisotropy of the stiffness tensor [17] and due to the shape of the NPs [9, 18].

On the other side, using atomistic simulations, Raman and Kara [19, 20] studied the vibrational density of state of metallic NPs. They showed that there is an enhancement in the vibrational density of states at low frequencies and an overall shift of the high frequency band beyond the top of the bulk phonons when decreasing the NP size. The effect of the capillary pressure induced by the surface of the NPs has also been exhibited on the vibrational density of state using atomistic numerical approaches [21, 22]. Besides the study of the vibrational density of states, severals studies have focused on few given vibration eigenmodes of NPs. Focusing on Raman active vibration modes, the elasticity theory showed a very good agreement with atomistic calculations in spherical germanium nanoparticle 23, 24. Recently, a breakdown of frequency-spectra scaling of respectively, silicon and zinc oxide nanoparticles have been evidenced using atomistic simulations and attributed to surface effects [14, 25, 26].

In this study, we would like to address three issues 
concerning the vibration properties of metallic NPs comparing the linear elasticity predictions and atomistic calculations.

First, we compare the vibration modes of metallic NPs calculated using either the elasticity theory or an atomistic approach based on an Embedded Atom Model (EAM). A similar study has been performed by Cheng et al. 23] in the case of germanium NPs. However, their elastic calculations did not take into account the anisotropy of the stiffness tensor. In this study, we show that taking into account this anisotropy in the elastic calculations improves the agreement between both approaches and that linear elasticity reproduces very well the vibration modes of NPs having a diameter of a few nanometers.

Second, it is well known, that the vibration properties of a NP containing $N$ atoms can be described by $3 N$ normal vibration modes. Therefore reducing the size of the NP also reduces the number of normal vibration modes. Elasticity, as for it, can not take into account this reduction because of its description using a continuum medium. Some studies include this reduction in elasticity theory by artificially limiting the number of modes 27]. In this study, we illustrate the reduction of the number of normal modes by showing the disappearance of certain modes while decreasing the NPs size.

Third and finally, while elasticity predicts that the frequency of a given normal mode scales as the inverse of a characteristic length of the NP, surface effects can alter this law [14, 25]. We analyze this scaling in the case of silver and gold spherical NPs and exhibit a characteristic size under which the frequency scaling break down. The breakdown of this scaling law is related to the surface stress and surface relaxation effects.

Section II describes the numerical method used for the elastic calculations, the atomistic model and the procedure used for comparing the two models. Section III addresses the three problems mentioned above. Most of the results presented here concern silver NPs, except for the last issue for which the case of gold NP has been considered.

\section{METHOD}

\section{A. Elastic Calculation}

\section{Solving the Navier equation with an anisotropic stiffness tensor}

The displacement fields $\vec{u}$ associated to each vibration mode are calculated in the scope of the linear elasticity theory by solving the Navier equation [28]:

$$
\rho \omega^{2} u_{i}+\sum_{j k l} \mathbf{C}_{i j k l} \frac{\partial^{2} u_{k}}{\partial x_{l} \partial x_{j}}=0
$$

where $\rho$ is the mass density, $\omega$ the frequency, $u_{i}$ the $i^{t h}$ component of the displacement fields $\vec{u}$ and $\mathbf{C}$ the fourth order stiffness tensor. This equation can be solved analytically for a spherical system in the particular case of an isotropic stiffness tensor [12]. In the case of an anisotropic stiffness tensor, we turn to a numerical solver. Using the scheme of Visscher et al. [29], we develop the displacement field components on a polynomial basis. Solving Eq. (1) then reduces to matrix algebra. We use in this study polynomials of order up to 20 to ensure the convergence of the method. Note that this parameter depends on the vibration mode of interest: high frequencies vibration modes commonly correspond to high spatial frequencies which require an extensive polynomial basis.

\section{Modes of interest and identification}

Severals experimental techniques enable the observation of acoustic vibration modes, the main ones being inelastic neutron scattering(INS) 30], Raman scattering, time-resolved pump-probe experiments and far-infrared absorption. Whereas the absence of selection rules in INS makes it sensitive to all vibrations, the three other cited experimental techniques only probe a few acoustic vibration modes. Therefore, among the very numerous calculated vibration modes, we are mainly interested in those observable by Raman scattering experiments. Selection rules for Raman scattering and far-infrared absorption for an isotropic solid sphere have been given by Duval[31]. Only the breathing (spheroidal, $\ell=0$ ) and quadrupolar (spheroidal, $\ell=2$ ) modes are Raman active. Note that time-resolved pump-probe experiments only detect the breathing modes of spherical NP 32].

The irreducible representations corresponding to all the vibration modes have been determined after the displacement fields have been calculated [15]. For a sphere made of a material having a cubic elasticity (such as silver or gold), the non-degenerate breathing mode transforms into an $\mathrm{A}_{1 \mathrm{~g}}$ vibration $\left(\mathrm{O}_{\mathrm{h}}\right.$ point group). The quadrupolar mode splits into $\mathrm{E}_{\mathrm{g}}$ and $\mathrm{T}_{2 \mathrm{~g}}$ vibrations 15,17 and the corresponding frequency splitting is large making the usage of elastic anisotropy a key factor for materials such as gold or silver.

\section{B. Atomistic Calculation}

\section{Calculation details}

We perform atomistic simulations using the EAM potentials developed by Clery and Roseto [33]. Table I reports structural and elastic properties calculated in the scope of this model for silver and gold using the program GULP 34, 35, 36].

The calculated elastic coefficients have been used as input in the Navier equation (11) in Sect. IIA in order to focus on the comparison of the models and eliminate 


\begin{tabular}{ccc}
\hline \hline & & \\
& silver & gold \\
& & \\
\hline & & \\
$\mathrm{a}$ & $4.078 \AA$ & $4.079 \AA$ \\
$C_{11}$ & $132.81(131)$ & $187.38(187)$ \\
$C_{12}$ & $97.47(97)$ & $154.40(155)$ \\
$C_{44}$ & $51.11(51)$ & $44.71(45)$ \\
\hline \hline
\end{tabular}

TABLE I: Cell parameter and elastic coefficients of $\mathrm{Ag}$ and $\mathrm{Au}$ calculated using the EAM parameters of Cleri and Rosato 33]. Elastic coefficients are expressed in GPa. Experimental data 37] for elastic coefficients are reported in parenthesis.

possible differences arising from slightly different elastic tensors.

Spherical NPs are designed with atoms initially placed on a perfect cubic close-packed crystal structure using the bulk cell parameters of the modeled material. Our NPs do not specifically reproduced NPs with magic number 38]. In addition, our construction procedure inevitably produces some steps and facets especially for very small NP. The total energy is then relaxed using a conjugate gradient algorithm: this procedure ensures that all vibration eigenfrequencies are real. Our model omits the possible existence of surface reconstructions and dangling bonds: these aspects could correctly be addressed using more sophisticated techniques (for instance DFT or tight binding). The dynamical matrix is then computed and diagonalized to obtain eigenfrequencies and eigenvectors. Eigenvectors are normalized for the usual scalar product in the real space of dimension $3 N$ with $N$ the number of atoms in the NP. In the following, we study spherical NPs from 19 to 2072 atoms corresponding to diameter ranging from $1.6 \mathrm{~nm}$ to 4.3 $\mathrm{nm}$ for silver NPs and from 10 to 2491 atoms corresponding to diameter ranging from $1.4 \mathrm{~nm}$ to $4.5 \mathrm{~nm}$ for gold NPs. The validity of the EAM potential for a 10 atoms $\mathrm{NP}$ is arguable and we admit that an ab-initio density functional calculation would be more appropriated in this case. However, in the following we compare the vibration properties of NPs on a large range of sizes. As a result, it does not seem judicious to use different atomistic models for different sizes since it would also result in a slight modification of the elastic stiffness tensor. As a matter of fact, though quantitative results for the smallest NPs would need a more accurate modeling, we believe that results described below are semi-quantitative in the sense that they give a good sketch of the vibration properties of NPs while reducing size.

In the following, we will refer to atomistic modes for vibration modes calculated in the framework of the atomistic EAM calculations.

\section{Modes identification}

While the linear elasticity theory assumes a continuous medium and thus provide a displacement field for each vibration mode, atomistic vibration modes are described by the data of each atom moves [24]. For this reason, and because the precise symmetry of the NPs shapes is not fixed when relaxing the energy, the symmetry analysis we did in the case of the elastic calculation becomes more troublesome in the case of atomistic modes. Instead, we prefer to project elastic modes on atomistic ones as it has already been done in the case of semi-conductor NPs [23]. The scalar product of the $p$ th elastic mode with the $q$ th atomistic mode reads:

$$
<\vec{u}_{p}^{\text {elas }} \mid \vec{u}_{q}^{\text {atomic }}>=\sum_{i \in \text { atoms }} \vec{u}_{p}^{\text {elas }}\left(\vec{R}_{i}\right) \cdot \vec{u}_{q, i}^{\text {atomic }}
$$

where $\vec{R}_{i}$ are the atomic positions in the atomistic model and $\vec{u}_{q, i}^{\text {atomic }}$ is the displacement of atoms $i$ located at position $\vec{R}_{i}$. Note that atomistic modes are normalized for this scalar product. We also normalize elastic modes so that:

$$
\sum_{q}<\vec{u}_{p}^{\text {elas }} \mid \vec{u}_{q}^{\text {atomic }}>^{2}=1
$$

A more relevant quantity is the sum of such squared projections of elastic modes onto atomistic ones for all degenerate elastic modes having the same irreducible representation and eigenfrequency[15]. More precisely, if $\Omega_{\text {elas }}$ is a set of such elastic modes, we define its projection on atomistic ones as:

$$
Q_{q}^{\Omega_{\text {elas }}}=\sqrt{\frac{\sum_{p \in \Omega_{\text {elas }}}<\vec{u}_{p}^{\text {elas }} \mid \vec{u}_{q}^{\text {atomic }}>^{2}}{\mathbb{N}_{\Omega_{\text {elas }}}}}
$$

where $\mathbb{N}_{\Omega_{\text {elas }}}$ is the number of modes in the set $\Omega_{\text {elas }}$. The denominator in Eq.(4) ensures the normalisation condition (similar to Eq.(3) ) for the projection of a set of elastic modes. The quantity $Q_{q}^{\Omega_{\text {elas }}}$ does not depend on the choice of degenerate elastic displacements. As such it is suitable for the comparison with atomistic calculations and will be used in the following.

\section{RESULTS}

\section{A. Elastic regime: Relevance of the anisotropy of the stiffness tensor}

\section{Mode Projection}

When surface effects are negligible, i.e. the NPs size is larger than a characteristic size which will be established below, the elasticity theory reproduces very well the atomistic calculations. 
Fig. 1 reports the projection of the set of fundamental quadrupolar $E_{g}$ and $T_{2 g}$ modes calculated by elasticity theory on atomistic modes for a $4.3 \mathrm{~nm}$ diameter silver spherical NP using Eq. (4). In order to show the relevance of the anisotropy of the stiffness tensor in elastic calculations, Fig. 1 reports the projection of the set of fundamental quadrupolar elastic modes calculated using both an isotropic and an anisotropic stiffness tensor. The anisotropic elastic calculations use the stiffness tensor calculated from the EAM atomistic model and reported in Tab. [. The isotropic calculation uses a stiffness tensor obtained using the 3D averaged sound velocities. Atomistic calculations intrinsically include the anisotropy of the elastic properties.

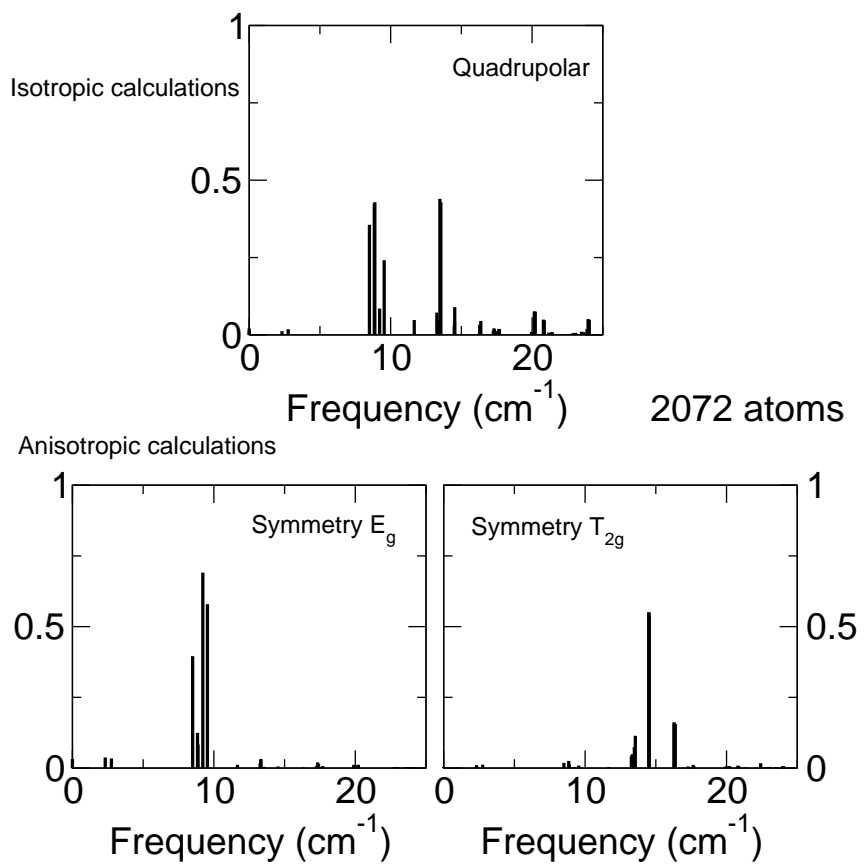

FIG. 1: (color online) Projection of the fundamental quadrupolar elastic vibration modes of a $4.3 \mathrm{~nm}$ diameter silver spherical NP, calculated using the linear elasticity theory on atomistic ones using the scalar product given in Eq. (2) and Eq. (4) . Results are reported for both isotropic (above) and anisotropic (below) calculations. For isotropic calculations, the quadrupolar elastic vibrations modes form a set of 5 degenerated modes with the same irreducible representations. In the anisotropic calculations, quadrupolar modes splits in two sets of modes of respective symmetry $E_{g}$ and $T_{2 g}$. Abscissa denotes atomistic modes using their frequency in $\mathrm{cm}^{-1}$.

In the isotropic elastic approximation the displacements of the 5 quadrupolar vibrations $(m=0, \pm 1, \pm 2)$ correspond to a single frequency. In the anisotropic elastic approximation, this degeneracy is partially lifted. As a result, 2 of these "quadrupolar" vibrations have the same frequency ( $\mathrm{E}_{\mathrm{g}}$ vibrations) and the 3 others have a different frequency ( $\mathrm{T}_{2 \mathrm{~g}}$ vibrations). It should be noted that the main effect of elastic anisotropy is to split the frequencies into 2 groups but that the corre- sponding displacements are almost unaffected [15]. Fig. 1 provides a simple way to check that taking into account anisotropy in the elastic calculations significantly improves the description. The quadrupolar modes from the isotropic elastic calculation have a significant projection onto atomistic modes over a much larger range than the corresponding anisotropic "quadrupolar" elastic modes. Therefore the anisotropic description is clearly an improvement. The $\mathrm{E}_{\mathrm{g}}$ vibrations have a significant projection over a narrow frequency range. For the $\mathrm{T}_{2 \mathrm{~g}}$ vibrations, the projections are significant over a larger frequency range, but this is probably due to the light mixing which occur in the elastic calculations between the $T_{2 g}$ branches coming from the isotropic quadrupolar and torsional $(\ell=3)$ modes 15$]$. This mixing is different within the atomistic approach which explains the appearance of an additional peak at higher frequency $\left(\sim 16.3 \mathrm{~cm}^{-1}\right)$.

In the following, elastic calculations always use the anisotropic stiffness tensor defined from Tab. [1]

\section{B. Size dependence and mode number reduction}

Following the work of Cheng et al. 223] we report in Fig. 2 the evolution of the projection of the fundamental breathing mode for different silver spherical NP sizes. This particular mode is the $\mathrm{A}_{1 \mathrm{~g}}$ vibration with a frequency close to the isotropic breathing mode and having the largest volume variation [15]. The projection of this elastic mode onto the atomistic ones is all the more peaked on a single atomic mode for big NPs. This observation agrees with the expectation that elasticity works well for large NPs for which the continuous medium approximation is more appropriated. Note that even for a very small NP, elasticity reproduces fairly well the atomistic modes. The same conclusion has been raised by Cheng et al. 23] while studying spherical germanium NPs.

Reducing the size of the NP diminishes its number of atoms. Thus, it also reduces the number of vibration modes. From Fig. 2, the breathing mode is evidenced even for the very small NP containing only 19 atoms. However, we expect that higher harmonics may disappear from the atomistic spectra while decreasing the NP size. In Fig. 3. we report the projection of the first overtone of the breathing mode $\left(\mathrm{A}_{1 \mathrm{~g}}\right.$ vibration with a frequency close to the first overtone of the isotropic breathing mode and having the largest volume variation) of silver spherical NPs on atomistic modes as a function of the NP size.

Contrarily to the fundamental breathing mode, the projection of the first overtone spreads over several atomistic modes for NPs made of 249 atoms and less. It is still possible to assign a main corresponding atomistic mode for a 249 atoms NP but no atomistic modes seem to describe the elastic mode for the 87 atoms NP. Instead, this mode projects onto several atomistic modes with a weak scalar product. For smaller NPs, it mainly projects onto the same atomistic mode as the fundamen- 


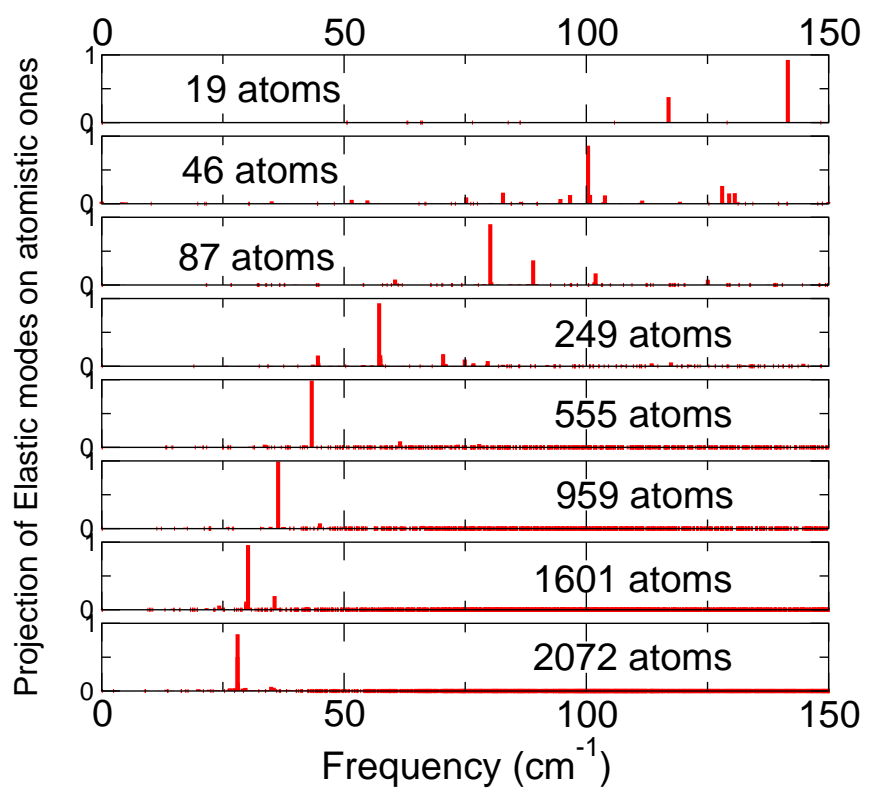

FIG. 2: (color online) Projection of the breathing vibration modes of silver spherical NP calculated using linear elasticity theory onto the atomistic modes using the scalar product given in Eq. (2). The number of atoms in the NP is reported on each graph. The frequency in $\mathrm{cm}^{-1}$ of the atomistic modes is used on the abscissa.

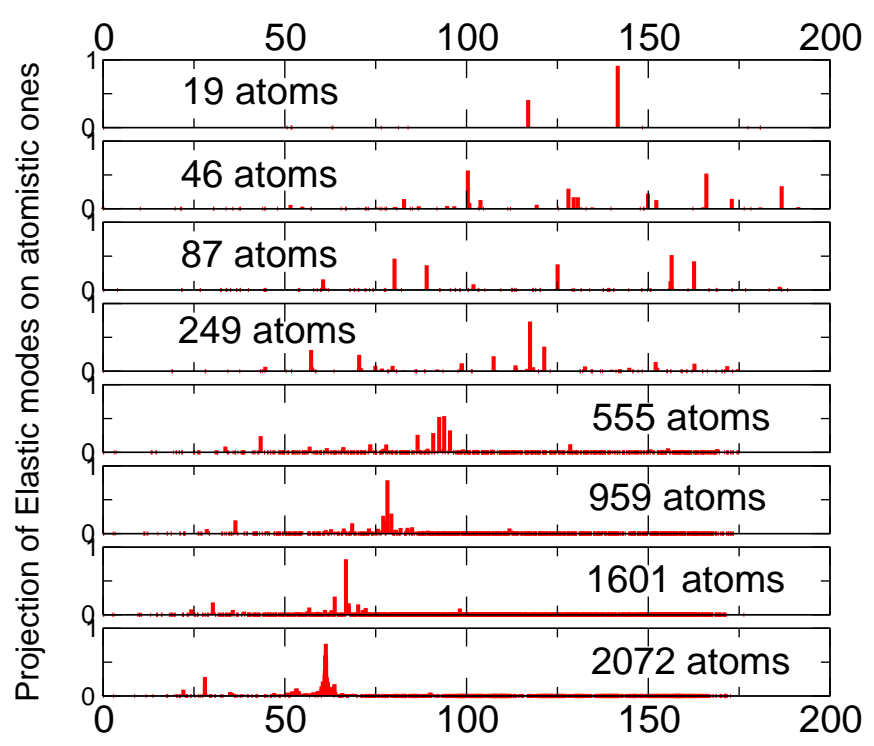

FIG. 3: (color online) Projection of the first overtone of the breathing mode of silver spherical NPs calculated using linear elasticity theory onto the atomistic modes using the scalar product given in Eq. (2). The number of atoms in the NP is reported on each graph. The frequency in $\mathrm{cm}^{-1}$ of the atomistic modes is used on the abscissa.

\section{Size dependence: breakdown of elasticity prediction}

\section{Power Law}

Using linear elasticity theory and from Eq. (11), the frequency of a given vibration mode of a solid sphere scales as $1 / R$ where $R$ is the radius of the sphere. This law which we will refer to as the Elastic Frequency Law (EFL) in the following, can be used to probe the validity of the elasticity theory. Recently, the validity of the EFL has been questioned for very small NP for which surface effects become non negligible 14, 25].

In this section, we investigate the validity of the EFL in the case of metallic NP. We thus report the frequency of a given atomistic mode as a function of $1 / N^{1 / 3}$ (proportional to the radius of the NP) with $\mathrm{N}$ the number of atoms in the NP. This atomistic mode is defined as the one on which the projection of elastic modes is maximum ignoring the relative values of this projection compared to the other modes. Figure 4 reports the frequencies of four selected atomistic modes for spherical silver NP as a function of $1 / N^{1 / 3}$. We first choose four elastic modes: one $\mathrm{E}_{\mathrm{g}}$ mode and one $T_{2 g}$ mode coming from the fundamental isotropic quadrupolar mode and two $\mathrm{A}_{1 \mathrm{~g}}$ modes coming from the fundamental and first overtone of the isotropic breathing mode. Then for each elastic mode and number of atoms $N$, we consider only the atomistic mode onto which the elastic mode has the largest projection and report its frequency as a function of $1 / N^{1 / 3}$. To identify the possible breakdown of the scaling law, we also plot the 

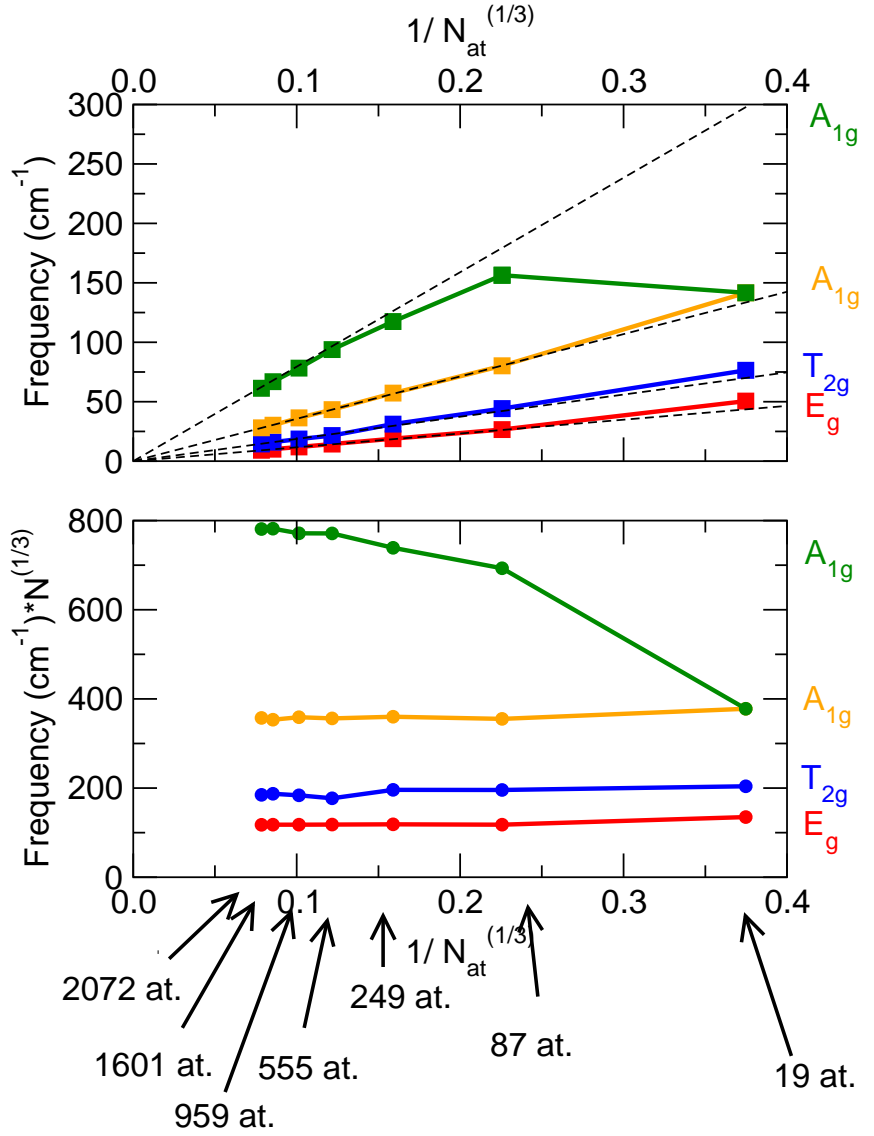

FIG. 4: (color online)(above) Frequencies of the atomistic modes onto which the $E_{g}$ and $T_{2 g}$ modes coming from the fundamental quadrupolar modes and the $\mathrm{A}_{1 \mathrm{~g}}$ modes coming from the fundamental (low frequency) and first overtone (high frequency) of the breathing mode preferentially project in spherical silver NPs as a function of the inverse of the number of atom at the power one third. Dashed lines correspond to frequencies calculated by the elasticity theory: the number of atoms is calculated from the ratio of the NP volume on the atomic volume computed from atomistic calculations. (below) Product of the frequency and the number of atoms at the power one third as a function of the inverse of the number of atoms at the power one third. Linear elasticity predicts horizontal straight lines.

product of the frequencies and $N^{1 / 3}$ as a function of $1 / N^{1 / 3}$. Note that the evaluation of the radius of a NP in the atomistic model may involve some technical issues regarding the precise definition of the surface, especially for small NPs: the surface is not exactly spherical and the position of the surface can be slightly different from the position of the surface atoms when taking into account atomistic radius. As a consequence, we prefer to report frequencies as a function of the unambiguous quantity $1 / N^{1 / 3}$.

Figure 4 shows that atomistic calculations agree well with the EFL for the atomistic quadrupolar modes and the atomistic fundamental breathing mode. Except for
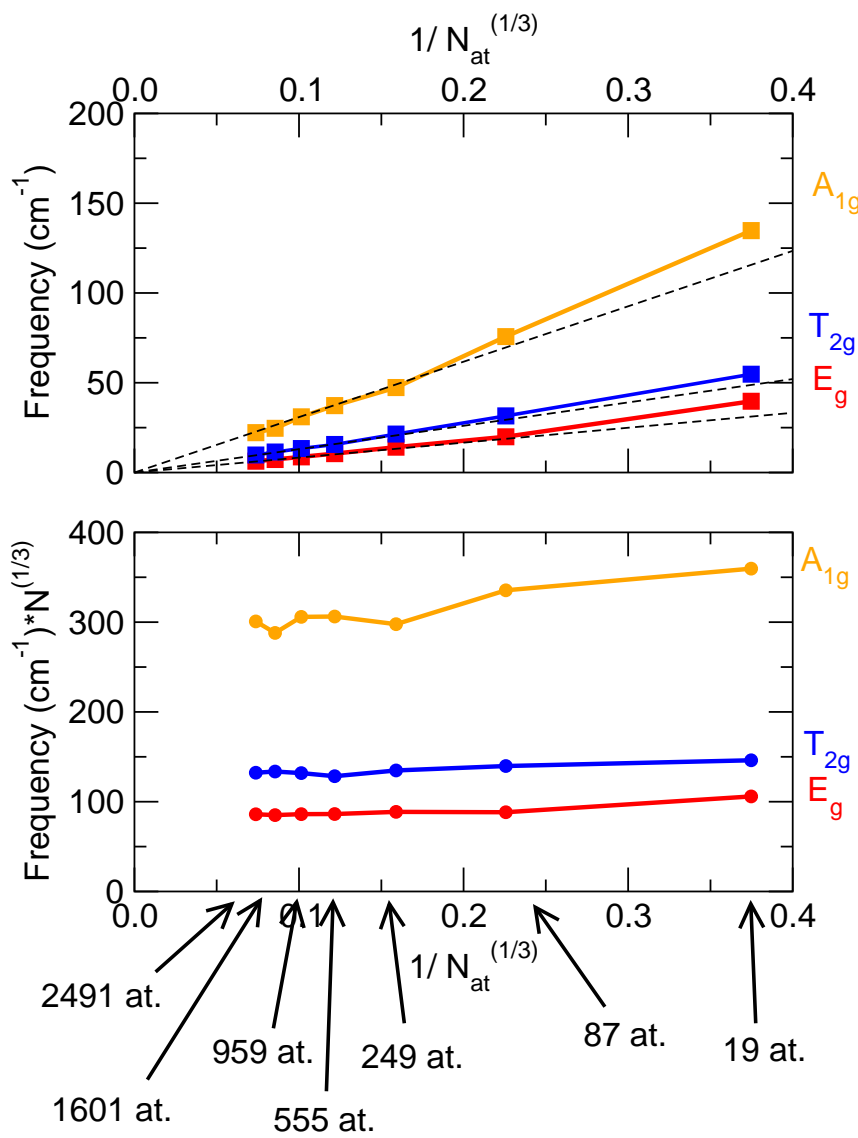

FIG. 5: (color online) (above) Frequencies of the atomistic modes onto which the $\mathrm{E}_{\mathrm{g}}$ and $\mathrm{T}_{2 \mathrm{~g}}$ modes coming from the fundamental quadrupolar modes and the $\mathrm{A}_{1 \mathrm{~g}}$ mode coming from the fundamental breathing mode preferentially project in spherical gold NPs as a function of the inverse of the number of atom at the power one third. Dashed lines correspond to frequencies calculated by the elasticity theory: the number of atoms is calculated from the ratio of the NP volume on the atomic volume computed from atomistic calculations. (below) Product of the frequency and the number of atoms at the power one third as a function of the inverse of the number of atoms at the power one third. Linear elasticity predicts horizontal straight lines.

the smaller NP with 19 atoms, for which the frequency differs slightly from the EFL, all the other points follow the EFL. The frequencies of the atomistic fundamental breathing mode and the two fundamental quadrupolar modes differ by about $6 \%\left(8 \mathrm{~cm}^{-1}\right)$ and $5 \mathrm{~cm}^{-1}$ and $6 \mathrm{~cm}^{-1}$ respectively for 19 atoms NP. Difference for bigger NP are smaller than $1 \mathrm{~cm}^{-1}$.

Concerning the first overtone of the breathing mode, Fig. 4 shows that the atomistic frequency starts to differ significantly from the EFL for less than about 250 atoms corresponding to a characteristic diameter of $2.2 \mathrm{~nm}$. This breakdown of the EFL could be related to surface effects[14] (we exclude at this point the case of the 19 atoms NP). Surface and surface relaxation effects thus do not affect equivalently the different vibration modes. 
The relaxation of the surfaces spreads on a characteristic size $\lambda$ of a few Angstroms under the surface[21]. We can reasonably expect that modes with a small wavelength (such as harmonics of isotropic modes) are more affected than longer wavelength ones (or fundamental mode) by the presence of the surface. Obviously this claim also depends on the relative volume affected by the surface relaxation compared to the unaffected volume in the NP: frequencies of optical modes or border zone phonon modes in a semi-infinite medium are not affected by the presence of the surface. Finally, for the 19 atoms NP, the atomistic first overtone of the breathing mode is identical to the atomistic fundamental breathing mode because of the reduction of the number of modes and symmetry arguments as already discussed in Sect. IIIB

In addition, we performed the same study for gold NP to investigate the dependence of our results on the material. Figure 5 reports the frequency of the fundamental quadrupolar and breathing atomistic modes for a spherical gold $\mathrm{NP}$ as a function of $1 / N^{1 / 3}$. While the atomistic quadrupolar modes reproduce the EFL fairly well, the atomistic fundamental breathing mode significantly shifts from the EFL at a characteristic size of about 87 atoms corresponding to a diameter of about $1.6 \mathrm{~nm}$ : the frequency of the atomistic fundamental breathing mode differs by about $18 \%\left(21 \mathrm{~cm}^{-1}\right)$ from the EFL for 19 atoms NP and by about $12 \%\left(8 \mathrm{~cm}^{-1}\right)$ for the 87 atoms NP. As in the case of silver NP, we attribute the breakdown of the EFL to surface relaxations effects. Note that our results suggest that surface effects seems to be more important in gold NPs than in silver NPs (for which the atomistic fundamental breathing mode reproduce the EFL fairly well except for the smallest studied $\mathrm{NP}$ ). This result is corroborated by the fact that surface relaxation in gold NP extends over a larger range than in silver [21, 39], and that the surface stress (to which surface relaxation is related) in gold is about two times the one in silver: this last result has been obtained for different surface orientations by atomistic simulations based on the EAM [40, 41] and on the Modified EAM 42].

In addition, to rule out the dependence of our results on the precise shape of the NP, we have also performed simulations on truncated cuboctahedron silver NPs: no significant deviation from the results presented here has been observed.

\section{CONCLUSION}

By comparing atomistic and linear elasticity calculations, we have exhibited the importance of the anisotropy of the stiffness tensor in elastic calculations. Especially, both atomistic and anisotropic elastic calculations lift the degeneracy of the quadrupolar modes. By projecting the elastic modes onto the atomistic ones, we evidenced the good agreement between the two models for the fundamental breathing mode even for very small NPs. These two results suggest the applicability of the linear elastic theory to less than $2 \mathrm{~nm}$ diameter silver NPs. However, we also evidenced the decreasing number of vibration modes and the breakdown of the EFL when reducing the size. We conclude, that the applicability of the linear elasticity depends on the considered vibration mode and the NP size. Our results suggest that the breakdown of elasticity occurs for bigger NPs for high harmonics rather than for fundamentals modes. Moreover, critical sizes depend on the material. Such breakdown to our knowledge, has never been evidence experimentally in any material because of the difficulty to synthesize NPs of diameter of less than $2 \mathrm{~nm}$ with a narrow size distribution or to perform single particle measurements on such objects. Our results suggest that the observation of the breakdown of the vibration frequency scaling in silver NPs will be hazardous, since it would imply some experiments on silver NP (or cluster) of about 20 atoms for the fundamental breathing and quadrupolar modes. This task though difficult, seems more reachable in the case of gold NP since it would require measurements on NPs with less than about 100 atoms. Finally, because the shape of very small NPs can hardly be described by a sphere, we have confirmed through simulations on truncated cuboctahedron silver NPs that our results do not strongly depend on the exact shape of the NPs.

\section{Acknowledgments}

NC thanks the support of the CNano GSO program and the PPF Grand Sud-Ouest.
[1] C. Q. Chen, Y. Shi, Y. S. Zhang, J. Zhu, and Y. J. Yan, Phys. Rev. Lett. 96, 075505 (2006).

[2] B. Wen, J. E. Sader, and J. J. Boland, Phys. Rev. Lett. 101, 175502 (2008).

[3] J. He and C. M. Lilley, Nanolett. 8, 1798 (2008).

[4] S. Cuenot, C. Frétigny, S. Demoustier-Champagne, and B. Nysten, Phys. Rev. B 69, 165410 (2004).

[5] M. T. McDowell, A. M. Leach, and K. Gall, NanoLett. 8, 3613 (2008).

[6] B. Palpant, H. Portales, L. Saviot, J. Lermé, B. Prével,
M. Pellarin, E. Duval, A. Perez, and M. Broyer, Phys. Rev. B 60, 17107 (1999).

[7] H. K. Yadav, V. Gupta, K. Sreenivas, S. P. Singh, B. Sundarakannan, and R. S. Katiyar, Phys. Rev. Lett. 97, 085502 (2006).

[8] H. K. Yadav, V. Gupta, K. Sreenivas, S. P. Singh, B. Sundarakannan, and R. S. Katiyar, Phys. Rev. Lett. 98, 029902(E) (2007).

[9] P.-M. Chassaing, F. Demangeot, N. Combe, L. SaintMacary, M. L. Kahn, and B. Chaudret, Phys Rev. B 79, 
155314 (2009).

[10] E. Ozbay, Science 311, 189 (2006).

[11] S. Maier, Plasmonics: Fundamentals and Applications (Springer, 2007).

[12] H. Lamb, Proc. London Math. Soc. 13, 187 (1882).

[13] H. Portales, L. Saviot, E. Duval, M. Fujii, S. Hayashi, N. D. Fatti, and F. Vallée, J. Chem. Phys. 115, 3444 (2001).

[14] N. Combe, P.-M. Chassaing, and F. Demangeot, Phys. Rev. B 79, 045408 (2009).

[15] L. Saviot and D. B. D. B. Murray, Phys. Rev. B 79, 214101 (2009).

[16] L. Saviot and D. B. Murray, Phys. Rev. Lett. 93, 055506 (2004).

[17] H. Portales, N. Goubet, L. Saviot, S. Adichtchev, D. B. Murray, A. Mermet, E. Duval, and M.-P. Pileni, PNAS 30, 14784 (2008).

[18] J. Burgin, P. Langot, A. Arbouet, J. Margueritat, J. Gonzalo, C. N. Afonso, F. Vallée, A. Mlayah, M. D. Rossell, and G. Van Tendeloo, NanoLett. 8, 1296 (2008).

[19] A. Kara and T. S. Rahman, Phys. Rev. Lett. 81, 1453 (1998).

[20] A. Kara and T. S. Rahman, Surf. Sci. Rep. 56, 159 (2005).

[21] R. Meyer, S. Prakash, and P. Entel, Phase Transitions 75, 51 (2002).

[22] R. Meyer, L. J. Lewis, S. Prakash, and P. Entel, Phys. Rev. B 68, 104303 (2003).

[23] W. Cheng, S. F. Ren, and P. Y. Yu, Phys. Rev. B 71, $174305(2005)$.

[24] N. Combe, J. R. Huntzinger, and A. Mlayah, Phys. Rev. B 76, 205425 (2007).

[25] F. Ramirez, P. R. Heyliger, A. K. Rappé, and R. G.
Leisure, Phys. Rev. B 76, 085415 (2007).

[26] F. Ramirez, P. R. Heyliger, A. K. Rappé, and R. G. Leisure, J. Acoust Soc. Am. 123, 709 (2008).

[27] A. Tamura and T. Ichinokawa, J. Phys. C : Solid State Phys 16, 4779 (1983).

[28] L. D. Landau and E. M. Lifshitz, Theory of elasticity (Pergamon Press, Oxford, 1986).

[29] W. M. Visscher, A. Migliori, T. M. Bell, and R. . A. Reinerr, J. Acoust. Am. Soc. 90, 2154 (1991).

[30] L. Saviot, C. H. Netting, D. B. Murray, S. Rols, A. Mermet, A.-L. Papa, C. Pighini, D. Aymes, and N. Millot, Phys. Rev. B 78, 245426 (2008).

[31] E. Duval, Phys. Rev. B 46, 5795 (1992).

[32] A. Arbouet, N. D. Fatti, and F. Vallée, J. Chem. Phys. 124, 144701 (2006).

[33] F. Cleri and V. Rosato, Phys. Rev. B 48, 22 (1993).

[34] J. Gale, JCS Faraday Trans. 93, 629 (1997).

[35] J. Gale, Phil. Mag. B 73, 3 (1996).

[36] J. Gale and A. Rohl, Mol. Simul. 29, 291 (2003).

[37] G. Simmons and H. Wang, Single Crystal Elastic Constants and Calculated aggregated Properties (MIT Press, Cambridge, 1971).

[38] T. P. Martin, Phys. Rep. 273, 199 (1996), ISSN 03701573.

[39] D. Y. Sun, X. G. Gong, and X.-Q. Wang, Phys. Rev. B 63, 193412 (2001).

[40] P. Gumbsch and M. S. Daw, Phys. Rev. B 44, 3934 (1991).

[41] G. J. Ackland, G. Tichy, V. Vitek, and M. W. Finnis, Phil. Mag. A 56, 735 (1987).

[42] J. Wan, Y. L. Fan, D. W. Gong, S. G. Shen, and X. Q. Fan, Modelling Simul. Mater. Sci. Eng. 7, 189 (1999). 